\title{
REGULATION DE LA SECRETION ANDROGENIQUE DU TESTITULE
}

\author{
G. Verhoeven
}

\author{
Legendo, Onderwijs en Navorsing, Gasthuisberg, 3000 Leuven, Belgique
}

\section{REGULATION OF TESTICULAR ANDROGEN SECRETION. Although LH} undoubtedly remains the major factor controlling testicular androgen production, there is increasing evidence for the existence of a complex network of paracrine and autocrine intratesticular factors capable of modulating the development, differenciation and activity of the Leydig cells. This review summarizes the present understanding of intratesticular factors affecting Leydig cell function. Attention has been focussed upon the identification of tubular agonist which might mediate the effects of FSH on the production of androgens by Leydig cells. The findings discussed illustrate that even for such a welldefined function there are many candidates, and that the major agonists remain to be characterized. Moreover, all our present knowledge is based on invitro data, and the in-vivo role of all these agonists remains to be evaluated. Key words : Testis, paracrine factors, Leydig cells, Sertoli cells, androgens, gonadotrophins. Andrologie, 1992, 2 : 3-7.

Chez le mâle, une production adéquate d'androgène est nécessaire non seulement au développement des organes de la reproduction, mais aussi au développement des caractères sexuels secondaires, au contrôle du comportement sexuel et au maintien de la spermatogénèse.

Quatre-vingt quinze pour cent de la production des androgènes est assurée par le testicule. Cet organe peut être subdivisé de façon anatomique et fonctionelle en deux compartiments: un compartiment interstitiel ayant une fonction endocrine et un compartiment tubulaire ayant une fonction exocrine: la spermatogénèse.

Le compartiment interstitiel et plus particulièrement les cellules de Leydig produisent des

Ce texte est le résumé d'un exposé donné à l'occasion de la réunion commune de la Société d'Andrologie de langue française et de la Deutsche Gesellshaft für Andrologie à Strasbourg (Novembre 1991). androgènes. Outre les cellules de Leydig, le compartiment interstitiel contient d'autres types cellulaires tels les macrophages, les cellules mésenchymateuses, les cellules endothéliales et les cellules péritubulaires myoïdes En effet, les cellules de Leydig occupent moins de la moitié du compartiment interstitiel et moins de $5 \%$ du volume testiculaire total.

Dans les cellules de Leydig, la testostérone est synthétisée à partir du cholestérol, grâce à une cascade complexe de réactions. Chaque étape de cette cascade représente un point potentiel où des mécanismes régulatoires peuvent exercer leur activité.

La production finale de testostérone dépend entre-autres de : l'apport (exogène ou endogène) de cholestérol ; du transport de ce cholestérol vers la membrane interne des mitochondries, de la conversion du cholestérol en pregnénolone par clivage de la chaîne latérale grâce à une enzyme à cytochrome $\mathrm{P} 450$ et de la conversion de précurseurs C21 en dérivées $\mathrm{C} 19$ par la $17 \alpha$ hydroxylase, $\mathrm{C} 17,20$ lyase, autre enzyme à cytochrome P450. En outre, dans des cellules immatures, une fraction importante de la testostérone peut être convertie en dérivés moins actifs $5 \alpha$ reduits par la $5 \alpha$ réductase et la $3 \alpha / \beta$ hydroxysteroid déshydrogénase.

L'activité androgénique des testicules est principalement sous le contrôle de la LH. En effet, après hypophysectomie, la production d'androgènes peut être rétablie par l'administration de LH ou d'hCG. En outre, si nous suivons la production des androgènes au cours du développement, nous pouvons distinguer chez l'homme, comme dans d'autres espèces, trois stades distincts d'activité: un stade foetal, un stade périnatal et un stade adulte. Chacun de ces stades est accompagné d'une augmentation de la production de la LH.

Les données récentes de la littérature montrent que l'action de la LH sur les cellules de Leydig est beaucoup plus complexe qu'on l'avait supposé d'une part. D'autre part une série d'autres hormones et parahormones peuvent influencer l'activité androgénique testiculaire.
De façon schématique, la fonction peut être modulée au moins à trois niveaux distincts.

Premièrement: la nature même de la population de cellules de Leydig est susceptible de changements au cours du développement : Au cours de la vie foetale, par exemple, on rencontre des cellules de Leydig différentes de leurs équivalents adultes tant sur le plan morphologique que fonctionnel. Morphologiquement, elles sont caractérisées entre autres par l'abondance des gouttelettes lipidiques et leur association en petits amas non vascularisés. Fonctionnellement, elles sont caractérisées par l'absence de phénomènes de désensibilisation après stimulation par la LH. Ces cellules foetales disparaissent, ou au moins leur proportion devient très faible, à l'âge adulte (10). Les mécanismes de contrôle responsables du remplacement de ces cellules foetales par des cellules adultes restent incompris.

Deuxièmement, dans certaines circonstances le nombre de cellules de Leydig peut être modulé : Au cours de la puberté, le nombre de cellules de Leydig augmente, d'abord par une différentiation de cellules souches (probablement des cellules mésenchymateuses) et ensuite par une multiplication des cellules de Leydig ellesmêmes (8). Les mécanismes responsables de ces modifications ne sont que partiellement connus. Non seulement la LH mais aussi la FSH, la prolactine, I'hormone de croissance et les androgènes influencent de façon directe ou indirecte ce processus de différentiation et de multiplication $(6,8)$.

Enfin, l'activité sécrétoire des cellules de Leydig peut être modifiée : La principale hormone responsable du contrôle des secrétions leydigiennes reste la LH. Elle interagit avec des récepteurs spécifiques qui ont été récemment caractérisés grâce aux techniques de biologie moléculaire. Ils contiennent 7 domaines transmembranaires et un long domaine extracellulaire responsable de l'interaction avec la LH (12). Cette interaction déclenche une série d'évènements dépendant de la production d'AMP cyclique mais probablement aussi d'autres messagers secondaires (4). De ces réactions résultent 
finalement une phosphorylation de diverses protéines intracellulaires et une stimulation de la stéroidogénèse.

Aux doses pharmacologiques, la $\mathrm{LH}$ ou l'hCG provoque une désensibilisation, c'est-àdire, une diminution de la sensibilité à la LH. Cette désensibilisation explique la réponse biphasique observée après injection d'hCG à forte dose (16).

Les mécanismes responsables de cette atténuation de la réponse stéroidogénique sont complexes. On peut en décrire plusieurs: I'occupation des récepteurs réduisant leur disponibilité en vue d'une restimulation; l'internalisation de ces récepteurs; peut-être un découplage entre récepteurs et protéines Gs et enfin plusieurs bloquages à divers niveaux de la stéroidogénèse. Le plus important de ces arrêts est situé au niveau de la $17 \alpha$ hydroxylase, 17, 20 lyase, il résulterait de l'augmentation de la production d'oestradiol après la stimulation primaire (7). Cet arrêt explique l'augmentation de $17 \alpha$ hydroxyprogesterone et la diminution de la production de testostérone observée après une dose désensibilisante d'hCG.

Mis à part ces effets biphasiques sur la production de testostérone, la LH exerce également des effets trophiques sur les cellules de Leydig. Elle augmente la production de plusieurs enzymes cruciales dont la $17 \alpha$ hydroxylase 17 , 20 lyase. Par exemple, des cellules de Leydig en culture dans un milieu dépourvu de LH, perdent leur capacité de produire de la testostérone, alors qu'elles produisent des précurseurs $\mathrm{C} 21$, telles la progestérone et la $17 \alpha$. hydroxyprogestérone en quantité importante. L'addition de concentrations minimales de LH au milieu est suffisante pour que les cellules gardent leur capacité normale de production androgénique (22).

Cependant, la LH n'est certainement pas le seul facteur capable d'influencer l'activité des cellules de Leydig. Ces cellules possèdent des récepteurs pour d'autres molécules circulantes comme la prolactine et l'IGF-I. De plus une série de facteurs locaux, responsables de régulations paracrines et autocrines, peuvent moduler les sécrétions androgéniques testiculaires $(7,15$, $17,26)$.

\section{Evidence de l'existence de mécanismes de contrôle intratesticulaire.}

Trois types d' arguments supportent l'hypothèse d'un contrôle local de la fonction endocrine du testicule: des observations basées sur l'étude de cellules testiculaires cultivées in vitro; des arguments d'ordre physiologique, et des faits en relation avec la clinique.

1. In vitro les cellules de Leydig répondent à différents médiateurs : Les cellules de Leydig isolées répondent non seulement à la $\mathrm{LH}$, mais également à toute une série d'autres substances, y compris plusieurs facteurs de croissance, la LHRH, la vasopressine, des catécholamines et des stéroides. En plus, les cellules de Leydig produisent, non seulement des stéroides, mais aussi plusieurs autres facteurs hormonaux et humoraux, comme opioides, CRF, oxytocine, IGF-I, etc. Des observations similaires ont été faites pour les cellules de Sertoli. De plus différents facteurs produits par un type cellulaire trouvent des récepteurs sur l'autre type. Ces arguments suggèrent l'existence de mécanismes humoraux de régulation entre les cellules de Sertoli et les cellules de Leydig. Cette hypothèse est corroborée par cette observation: pour la majorité de ces substances, les concentrations requises pour moduler la fonction leydigienne dépassent considérablement les taux sériques observés (26).

2. Plusieurs observations physiologiques supportent l'hypothèse de l'existence de mecanismes de contrôle locaux: Au sein du testicule, la testostérone elle-même agit comme une parahormone, plutôt que comme une hormone. En effet, les concentrations intratesticulaires de testostérone dépassent considérablement les valeurs sériques. Une concentration élevée de testostérone semble d'ailleurs être nécessaire au maintien de la spermatogénèse. Dès lors, I'existence de mécanismes locaux contrôlant cette concentration intratesticulaire est vraisemblable. Ces mécanismes ne sont pas nécessairement les mêmes que ceux qui contrôlent la concentration sérique des androgènes.

Un des arguments les plus convainquants en faveur d'une communication entre les compartiments tubulaire et interstitiel concerne les effets de la FSH sur la production androgénique, principalement au cours de la puberté.

Le prétraitement par la FSH in vivo de rats hypophysectomisés augmente considérablement la réponse des cellules de Leydig isolées à une stimulation aigue par la LH. Dans ce contexte, un prétraitement à la FSH est nettement plus efficace qu'un prétraitement à la LH (20).

Etant donné que les récepteurs à la FSH se trouvent exclusivement localisés dans le compartiment tubulaire (14), nous devons conclure que le compartiment tubulaire doit produire un ou plusieurs médiateurs secondaires responsables des effets de la FSH sur les cellules de Leydig.

D'autres arguments plaident en faveur de l'existence de mécanismes locaux de régulation. Ainsi une perturbation locale ou unilatérale de la spermatogénèse (induite par exemple par un échauffement ou une cryptorchidie unilatérale) produit des modifications dans la morphologie et la fonction des cellules de Leydig limitées au testicule ou à la région testiculaire affectée. Ces effets ne peuvent s'expliquer par des variations dans les concentrations en gonadotrophines car elles affecteraient indifféremment les deux testicules (5).

Les cellules de Leydig situées à proximité des tubules subissent des changements cycliques dans leur dimension et leur morphologie. Ces changements sont en relation avec les stades de la spermatogénèse. De nouveau, cette synchronisation suggère l'existence de communications locales entre les compartiments tubulaire et interstitiel (2).

3. Quelques observations cliniques supportent, elles aussi, la notion de mécanismes de contrôle intratesticulaires : Un réajustement de l'axe hypophyso-testiculaire peut être observé après une perturbation profonde de la spermatogénèse, comme par exemple au cours d'une chimiothérapie pour maladie de Hodgkin. En effet, chez ces malades on peut parfois noter des taux normaux d'androgènes, accompagnés d'une élévation de la $\mathrm{LH}$. Néanmoins, la réponse à l'hCG est normale, suggérant que la capacité stéroidogénique des testicules n'est pas affectée. Une hypothèse plausible serait donc que, dans ce type de situation, le compartiment tubulaire produit des facteurs locaux qui diminuent la sensibilité des cellules de Leydig à la LH (19).

La testotoxicose familiale, maladie héréditaire, est caractérisée par une puberté précoce secondaire à une activation prématurée des cellules de Leydig. Cette activation se produit apparemment indépendamment des gonadotrophines. Par conséquent on suggérait l'existence de mécanismes activateurs locaux. Bien qu'on ne puisse formellement exclure cette possibilité, des données récentes semblent indiquer qu'un médiateur sérique de nature inconnue pourrait être responsable de ce syndrome rare (11).

En conclusion, cette série d'arguments plaident en faveur de l'existence de mécanismes de contrôle locaux.

\section{Identification des parahormones testiculaires.}

De façon schématique, au moins trois approches expérimentales sont utilisables pour 
identifier le ou les médiateurs produits sous l'influence de la FSH et responsables de la production stéroidienne des cellules de Leydig.

Premièrement, on peut raisonablement postuler qu'un médiateur produit dans le compartiment tubulaire doit obligatoirement passer par le liquide interstitiel pour atteindre les cellules de Leydig. Par conséquent, on peut vérifier si le liquide interstitiel contient des substances capables de moduler la fonction des cellules de Leydig et si elles sont absentes de la circulation périphérique. Plusieurs auteurs ont aussi pu démontrer que le liquide interstitiel contient un ou plusieurs facteurs augmentant la steroidogénèse de façon dose-dépendante, et ceci même en présence d'hCG $(9,18)$. En outre, la production de ces facteurs parait stimulée par certains processus responsables d'une interruption de la spermatogénèse (p.ex. I'induction d'une cryptorchidie). A ce jour, la nature du ou des facteurs responsables de ces effets est inconnue. Mais il s'agirait de protéines d'un poids moléculaire de plus de 30.000 .

Une deuxième approche expérimentale permettant d'étudier les effets de facteurs locaux sur la fonction des cellules de Leydig est la réalisation de cocultures. Les productions stéroidiennes sous l'influence des gonadotrophines sont comparées dans des monocultures de cellules de Leydig ou de cellules de Sertoli avec celles dans des cocultures de ces mêmes types cellulaires $(15,24)$

Ainsi que le montre la fig. 1, après stimulation par la LH, les productions de précurseurs $\mathrm{C} 21$ et d'androgènes (androsténedione et testostérone) observées dans des cocultures sont beaucoup plus importantes que celles observées dans des monocultures de cellules de Sertoli ou de cel-

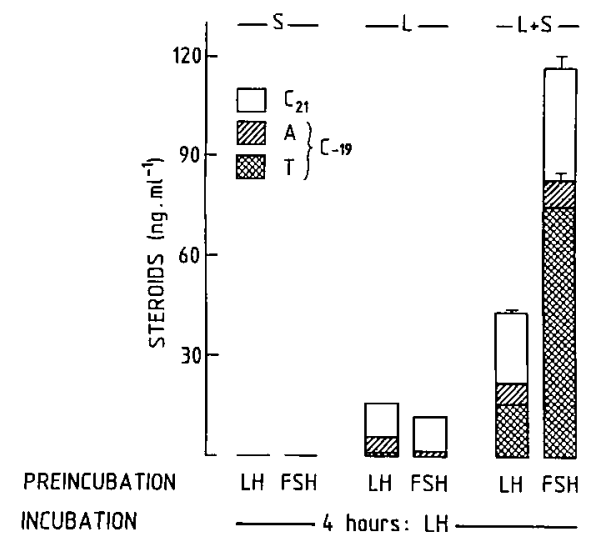

Fig. 1 : Production d'androgènes (testosterone et androsténédione) et de stérö̈des-C21 (progestérone, $17 \alpha$-hydroxyprogesterone et $20 \alpha$ hydroxy-pregn-4-en-3-one) par des cultures de cellules de Sertoli ( $S$ ), de cellules de Leydig (L) ou des cocultures $(L+S)$, prétraitées pendant 3 jours avec oFSH ou oL.H $(10 \mathrm{ng} / \mathrm{ml})$, puis stimulées pendant 4 heures avec $0 \mathrm{LH}(100 \mathrm{ng} / \mathrm{ml})$. lules de Leydig. En outre, un prétraitement, in vitro, avec la FSH n'a pas d'influence sur la capacité stéroidogénique des cellules de Leydig isolées mais augmente significativement la capacité stéroidogénique des cocultures. Finalement on peut noter qu'il y a dans les cocultures, une stimulation sélective de la production des androgènes et principalement de la testostérone.

Sans entrer dans les détails, vu la complexité des mécanismes moléculaires, on peut écrire que dans des cocultures, un prétraitement par la FSH accroît la concentration en récepteurs à la $\mathrm{LH}$ (15) et engendre des effets positifs non seulement au niveau de la production de précurseurs androgéniques, mais aussi au niveau de la conversion de ces précurseurs en testostérone (24).

Les effets positifs de la FSH dans des cocultures ne requièrent pas un contact direct entre les cellules de Leydig et les cellules de Sertoli ainsi que démontré par les systèmes de culture à deux chambres. Ces effets résultent donc d'effets dûs à des substances diffusibles en d'autres mots à des facteurs paracrines (24).

Par une troisième approche expérimentale d'éventuels facteurs paracrines seraient identifiés et caractérisés dans des milieux de culture conditionnés, issus soit de cellules isolées comme des cellules de Sertoli, soit de tubules testiculaires. Cette approche a conduit, dans plusieurs laboratoires, à l'identification partielle de diverses substances pouvant jouer un rôle paracrine $(1,22)$.

Dans notre laboratoire, nous avons surtout tenté d'identifier les médiateuts responsables des effets indirects de la FSH sur les cellules de Leydig. Nous avons pu isoler dans des milieux de culture conditionnés de cellules de Sertoli, plusieurs candidats. Un d'entre eux, que nous avons baptisé "Sertoli cell factor", est une protéine d'un poids moléculaire compris entre 10000 et 30000 . Cette molécule augmente la production d'androgènes par les cellules de Leydig même en présence d'une concentration optimale d'hCG. Le facteur semble être produit par plusieurs espèces animales et agit sur des cellules de Leydig de rat, de souris, et humaines. La production de "Sertoli cell factor" est considérablement augmentée par la FSH et par les cocultures avec des cellules peritubulaires. Par contre la production n'est pas influencée par les androgènes $(22,23)$.

Le "Sertoli cell factor" est un candidat valable comme médiateur des effets de la FSH, mais il reste à le purifier pour mieux en comprendre la signification physiologique.
Une autre substance, dont la nature est connue, pourrait jouer un rôle dans la communication entre le compartiment tubulaire et le compartiment interstitiel: c'est l'IGF-I. L'IGF-I est certainement produit par les cellules de Sertoli et stimule, au moins dans certaines circonstances, la fonction des cellules de Leydig $(3,15)$. Nous avons tenté d'étudier le rôle éventuel de l'IGF-I dans des cocultures de cellules de Sertoli et de cellules de Leydig en ajoutant des concentrations croissantes d'anticorps spécifiques anti-IGF-I et d'anticorps de contrôle (24). Dans ces conditions, l'anti-IGF-I provoque une chute importante et dose-dépendante de la production d'androgènes dans les cocultures. Cet effet n'est pas observé avec un anticorps de contrôle. Néanmoins, l'antiIGF-I diminue non seulement la production d'androgènes dans des cocultures, mais aussi dans des monocultures de cellules de Leydig. Donc, premièrement, I'IGF-I est un facteur indispensable pour maintenir la production d'androgènes dans des cellules de Leydig cultivées in vitro, et deuxièmement, les cellules de Leydig elles-mêmes produisent de l'IGF-I qui agit alors de façon autocrine. Par conséquent, la question persiste: I'IGF-I peut-il être considéré comme un médiateur des effets positifs et FSH-dépendants observés en coculture?

Il n'y a pas une réponse définitive à cette question mais deux types d'arguments plaident en faveur de cette hypothèse. D'abord, on observe que les taux d'IGF-I produits par des cocultures de cellules de Leydig et de cellules de Sertoli sont plus hauts que la somme de ceux sécrétés par les monocultures individuelles (3). Cette observation suggère des interactions positives et réciproques entre ces deux types de cellules. De plus, la FSH augmente non seulement la production d'IGF-I par des cellules de Sertoli, mais elle provoque simultanément une forte diminution de la sécrétion d'une protéine de liaison, spécifique pour ce facteur de croissance. Logiquement cette combinaison d'effets doit provoquer une stimulation accrue d'IGF-I libre, stimulation génératrice d'une augmentation de la production d'androgènes (3).

Le "Sertoli cell factor" et l'IGF-I sont-ils les seuls médiateurs des effets de la FSH sur les cellules de Leydig ou tout au moins les plus importants? Cela semble plutôt improbable. En effet, récemment nous avons effectué une série d'expériences utilisant des cellules de Leydig tumorales (H-540). Ces cellules ont perdu leur sensibilité non seulement à la LH mais aussi à l'IGF-I et au "Sertoli cell factor". Néanmoins, elles restent 
sensibles à l'effet stimulant et FSH-dépendant observé en coculture avec des cellules de Sertoli. Ainsi que nous l'avions observé avec les cellules de Leydig nontumorales, la réponse ne dépend pas de contacts immédiats avec des cellules de Sertoli. Elle doit donc être le fait d'un facteur paracrine inconnu à ce jour (25).

Jusqu'à présent les facteurs paracrines décrits exercent un effet positif sur les cellules de Leydig, et agissent comme médiateurs des effets de la FSH. Cependant plusieurs laboratoires ont décrit des facteurs tubulaires et/ou interstitiels inhibant la production d'androgènes. Certains de ces facteurs ont une identité biochimique bien connue, d'autres restent à être caractérisés $(21,26)$.

Tableau 1 : Molécules d'origine testiculaire à effets paracrines ou autocrines sur les cellules de Leydig

\begin{tabular}{|c|c|c|c|}
\hline & & SOURCE & $\begin{array}{c}\text { EFFET } \\
\text { MAJEUR }\end{array}$ \\
\hline $\begin{array}{l}\text { Hormones } \\
\text { peptidiques }\end{array}$ & $\begin{array}{l}\text { angiotensine II } \\
\text { facteur atrial natriurétique } \\
\text { oxytocine } \\
\text { vasopressine }\end{array}$ & $\begin{array}{c}(\mathrm{L}, \mathrm{G}) \\
5 \mathrm{I}^{\circ} \\
(\mathrm{S}, \mathrm{L}) \\
(\mathrm{I})\end{array}$ & $\begin{array}{l}- \\
+ \\
\pm \\
\pm\end{array}$ \\
\hline Stéroides & $\begin{array}{l}\text { androgènes } \\
\text { cstrogènes }\end{array}$ & $\begin{array}{c}(\mathrm{L}) \\
(\mathrm{L}, \mathrm{S})\end{array}$ & . \\
\hline $\begin{array}{l}\text { Facteurs de } \\
\text { croissance }\end{array}$ & $\begin{array}{l}\text { activine } \\
\text { inhibine } \\
\text { bFGF } \\
\text { IGF-I } \\
\text { TGF } \alpha \\
\text { TGF } \beta\end{array}$ & $\begin{array}{c}(\mathrm{S}, \mathrm{L}) \\
(\mathrm{S}, \mathrm{L}) \\
(\mathrm{S}) \\
(\mathrm{S}, \mathrm{P}, \mathrm{L}) \\
(\mathrm{S}, \mathrm{P}, \mathrm{L}) \\
(\mathrm{S}, \mathrm{P})\end{array}$ & $\begin{array}{l}- \\
+ \\
- \\
+ \\
\pm \\
-\end{array}$ \\
\hline $\begin{array}{l}\text { Releasing } \\
\text { factors }\end{array}$ & $\begin{array}{l}\text { LHRH } \\
\text { CRF }\end{array}$ & $\begin{array}{c}(\mathrm{I}) \\
(\mathrm{L}, \mathrm{G})\end{array}$ & $\begin{array}{l} \pm \\
-\end{array}$ \\
\hline Cytokines & $\begin{array}{l}\text { IL-1 } \\
\text { IL-2 } \\
\text { IFN- } \\
\text { TNF }\end{array}$ & $\begin{array}{l}(\mathrm{S}, \mathrm{M}) \\
(\mathrm{I}) \\
(\mathrm{I}) \\
(\mathrm{M})\end{array}$ & $\begin{array}{l} \pm \\
- \\
- \\
\pm\end{array}$ \\
\hline Divers & $\begin{array}{l}\text { agonistes B-adrénergiques } \\
\text { bradykinine } \\
\text { histamine } \\
\text { octadecaneuropeptide } \\
\text { substance P }\end{array}$ & $\begin{array}{l}(\mathrm{I}) \\
(\mathrm{I}) \\
(\mathrm{I}) \\
(\mathrm{L}) \\
(\mathrm{L})\end{array}$ & $\begin{array}{l}+ \\
+ \\
+ \\
? \\
+\end{array}$ \\
\hline $\begin{array}{l}\text { Partiellement } \\
\text { caractérisés }\end{array}$ & $\begin{array}{l}\text { Facteur cellulaire de Sertoli } \\
\text { Facteur tubulaire } 40-50 \mathrm{kD} \\
\text { Facteur tubulaire } 30-100 \mathrm{kd} \\
\text { Inhibiteur TM4 } \\
\text { h SCSP-80 }\end{array}$ & $\begin{array}{c}(\mathrm{S}) \\
(\mathrm{S}) \\
(\mathrm{S}) \\
(\mathrm{TM} 4) \\
(\mathrm{S})\end{array}$ & $\begin{array}{l}+ \\
- \\
- \\
- \\
+\end{array}$ \\
\hline
\end{tabular}

La source probable des agonistes intratesticulaires est indiquée entre parenthèses. $G$; cellules germinales ; I : source inconnue ; $L$; cellules de Leydig ; $\mathrm{M}$ : macrophages ; $\mathrm{P}$ : cellules péritubulaires ; $\mathrm{S}$ : cellules de Sertoli

Afin d'illustrer la complexité du problème, le tableau I résume les facteurs connus et partiellement caractérisés, capables de modifier la fonction des cellules de Leydig (in vitro au moins), et produits par le testicule. Cette liste de facteurs paracrines et autocrines potentiels comprend des hormones peptidiques, des stéroides, des facteurs de croissance, des "releasing-factors", des cytokines, des neurotransmetteurs et des peptides encore mal caractérisés. L'origine exacte de ces molécules n'est pas toujours connue. Un grand nombre de facteurs sont sécrétés par les cellules de Sertoli, mais d'autres proviennent probablement des macrophages, des cellules péritubulaires, des cellules germinales ou des cellules de Leydig elles-mêmes. En outre, l'effet de plusieurs de ces médiateurs sur les cellules de Leydig peut différer selon les conditions exactes d'expérimentation: le degré de maturation des cellules de Leydig, la durée de l'incubation, la présence simultanée d'autres substances comme la LH... etc.

Enfin, il ne faut oublier que la production de la majorité de ces facteurs paracrines et autocrines sont à leur tour controlés, soit par les gonadotrophines soit par d'autres substances. Cette régulation peut déterminer le rôle ultime des facteurs concernés. Le TGFB par exemple peut être produit par les cellules de Sertoli et les cellules péritubulaires et ce facteur de croissance a surtout des effets négatifs sur la production d'androgènes, mais dans des cellules de Sertoli la production de TGFB est inhibée par la FSH. Par conséquent, on peut imaginer que les effets positifs de la FSH sur la stéroidogénèse sont, au moins en partie, le résultat de l'inhibition de la production de TGF $B$ (13).

En conclusion, la LH reste sans doute la principale hormone responsable du contrôle de l'activité des cellules de Leydig. L'action de la LH est complexe et inclut des effets aigus, des effets de désensibilisation et des effets trophiques. Ces effets peuvent être modulés par d'autres médiateurs et notamment par de nombreux facteurs produits par le testicule même. $\mathrm{Ce}$ travail a pour but de montrer l'importance de la littérature qui s'est accumulée pendant les dernières années au sujet des mécanismes locaux responsables des effets sur la sécrétion androgénique. L'identification de facteurs tubulaires secrétés sous l'influence de la FSH et controlant la production des androgènes est l'objet de nos travaux personnels. Pour une telle fonction bien définie, plusieurs candidats existent, mais les plus importants restent à être identifiés. Enfin, presque toutes les connaissances actuelles sont basées sur des expériences in vitro. Des efforts considérables et des techniques nouvelles seront probablement nécessaires pour évaluer le rôle exact de tous ces facteurs locaux in vivo.

\section{REFERENCES}

1 - Benahmed M, Grenot C, Tabone E, Sanchez P, Morera AM: FSH regulates cultured Leydig cell function via Sertoli cell proteins: an in vitro study. Biochem. Biophys. Res. Comm., 1985,132:729.
2- Bergh A : Paracrine regulation of Leydig cells by the seminiferous tubules. Int. J. Androl., 1983,6:57.

3 - Cailleau J, Vermeire S, Verhoeven G. : Independent control of the production of insulinlike growth factor I and its binding protein by cultured testicular cells. Mol. Cell. Endocrinol., 1990,69:79.

4 - Cooke BA. : Is cyclic AMP an obligatory second messenger for luteinizing hormone ? Mol. Cell. Endocrinol., 1990,69:C11.

5 - de Kretser DM. : Sertoli cell-Leydig cell interaction in the regulation of testicular function . Int. $J$. Androl., 1982, Supplement 5: 11.

6 - Dombrowicz D. : Contrôle hypophysaire du développement testiculaire chez le rat: aspects biochimiques et histologiques. Thèse de doctorat, 1991, Liège, Belgium.

7. Dufau ML. : Endocrine regulation and communicating functions of the Leydig cell. Ann. Rev. Physiol., 1988, 50:483.

8 - Hardy MP, Zirkin BR, Ewing LL. : Kinetic studies on the development of the adult population of Leydig cells in testes of the pubertal rat. Endocrinology, 1989, 124:762.

9 - Hedger MP, Robertson DM, de Kretser DM, Risbridger GP. : The quantification of steroidogenesis-stimulating activity in testicular interstitial fluid by an in vitro bioassay employing adult rat Leydig cells. Endocrinology., 1990,127:1967.

10 - Kerr JB. : The fate of fetal Leydig cells in the developing rat testis. Serono Symposia Publications, 1988;50:175.

11 - Manasco PK, Girton ME, Diggs RL, Doppman J, Feuillan PP, Barnes KM, Cutler GB, Loriaux DL, Albertson BD. : A novel testis-stimulating factor in familial male precocious puberty. $\mathrm{N}$. Engl. J. Med, 1991, 324:227.

12 - Mc Farland KC, Sprengel R, Phillips HS, Kohler M, Rosulblit N, Nikolics K, Segaloff DL, Seeburg P. : Lutropin-choriogonadotropin receptor: an unusual member of the $G$ protein-coupled receptor family. Science, 1989,245:494.

13 - Morerar AM, Chauvin MA, Feige JJ, Guillaumot P, Keramidas M, Maudreit C, Sordvillet C, Benahmed $\mathrm{M}$. : Interactions between systemic hormones and local growth factors in the control of the testis function: the example of gonadotropins and transforming growth factor $B$. Serono Symp. Publ. 1990,70:191.

14 - Orth 1, Christensen AK, : Localization of 125 I labeled FSH in the testes of hypophysectomizedorats by autoradiography at the light and electron microscope levels. Endocrinology, 1977,101:262.

15 - Saez JM, Avallet O, Naville D, Perrard-Sapori MH, Chatelain PG. : Sertoli-Leydig cell communications. In : "Regulation of testicular function", Ewing LL et Robaire B eds. Annals of the New York Academy of Sciences, 1989,564:210.

16 - Saez JM, Forest MG. : Kinetics of human chorionic gonadotropin-induced steroidogenic response of the human testis. J. Clin. Endocrinol. Metab., 1979, 49:278. 
17 - Sharpe RM. : Intratesticular control of steroidogenesis . Clin. Endocrinol., 1990,33:787.

18 - Sharpe RM, Cooper I. : Intratesticular secretion of a factor(s) with major stimulatory effects on Leydig cell testosterone secretion in vitro. Mol. Cell. Endocrinol, 1984,37: 159.

19 - Tsatsoulis A, Whitehead E, John J, Shalet SM, Robertson WR : The pituitary Leydig cell axis in men with severe damage to the germinal epithelium. Clin. Endocrinol, 1987,27:683.

20 - Van Beurden WMO, Roodnat B, de Jong F, Mulder E, van der Molen HJ. : Hormonal regulation of LH stimulation of testosterone production in isolated Leydig cells of immature rats: the effect of hypophysectomy, FSH, and estradiol 17B. Steroids, 1976,28:847.

21 - Verhoeven G . : Local control systems within the testes. Baillières Clinical Endocrinology and Metabolism. The Testis de Kretser, ed. 1992, sous presse.

22 - Verhoeven G., Cailleau J. : A factor in spent media from Sertoli cell-enriched cultures that stimulates steroidogenesis in Leydig cells. Mol. Cell. Endocrinol, 1985,40:57.

23 - Verhoeven G., Cailleau : J. A Leydig cell stimulatory factor produced by human testicular tubules. Mol . Cell . Endocrinol, 1987,49: 137.
24 - Verhoeven G., Cailleau J. : Influence of coculture with Sertoli cells on steroidogenesis in immatur rat Leydig cells. Mol. Cell. Endocrinol. 1990,71:239.

25 - Verhoeven G, Cailleau J. : Rat tumor Leydig cells as a test system for the study of Sertoli cell factors that stimulate steroidogenesis. J. Androl., 1991,12:9.

26 - Verhoeven G, Cailleau J, Swinnen K, Heyns W : Local interactions between tubular and interstitial comparment in the testis. In A. Isidorni, A. Fabri, ML Dufau eds : Hormonal communicating events in the testis. Serono Symposia Publications, 1990,70:149.
RESUME : Bien que la LH reste le principal facteur responsable de la régulation de la sécrétion androgénique du testicule, le développement, la différentiation et l'activité des cellules de Leydig sont également modulés par toute une série de facteurs intratesticulaires. Dans cet article nous avons tenté de donner un aperçu de ce contrôle local (régulations paracrines et/ou endocrines) de la fonction des cellules de Leydig. Nos recherches ont porté sur l'identification de facteurs tubulaires qui pourraient moduler les effets de la FSH sur la production des androgènes. Les données présentées montrent l'existence de nombreux candidats à cette fonction. La plupart de ces molécules agonistes doivent encore être caractérisées. De plus leur rôle exact reste à être évalué in vivo. Mots clés : testicule, facteurs paracrines, cellules de Leydig, cellules de Sertoli, androgènes, gonadotrophines. Andrologie, 1992, 2 : 3-7. 